\title{
ON THE RADIAL LIMITS OF ANALYTIC AND MEROMORPHIC FUNCTIONS
}

BY

\author{
J. S. HWANG ${ }^{1}$
}

\begin{abstract}
Early in the fifties, A. J. Lohwater proved that if $f(z)$ is analytic in $|z|<1$ and has the radial limit 0 almost everywhere on $|z|=1$, then every complex number $\zeta$ is an asymptotic value of $f(z)$ provided the $\zeta$-points satisfy the following Blaschke condition: $\sum_{k=1}^{\infty}\left(1-\left|z_{k}\right|\right)<\infty$, where $f\left(z_{k}\right)=\zeta, k=1,2, \ldots$ We may, therefore, ask under the hypothesis on $f(z)$ how many complex numbers $\zeta$ are there whose $\zeta$-points can satisfy the Blaschke condition. We show that there is at most one such number and this one number phenomenon can actually occur if the number is zero.
\end{abstract}

1. Introduction. Examples have been given by N. Lusin and J. Privaloff [6, p. 185] that there are nonconstant functions $f(z)$, analytic in the unit disk $D:|z|<1$, for which the radial limit $f\left(e^{i \theta}\right)=\lim _{r \rightarrow 1} f\left(r e^{i \theta}\right)$ is zero almost everywhere on $C$ : $|z|=1$. Following A. J. Lohwater [5, p. 79], we shall call functions of this kind to be of the class (LP).

Let $f(z)$ be a function defined in $D$ and let $\zeta$ be a complex number. The set of all points $\left\{z_{k}\right\}$ in $D$ is called the $\zeta$-points of $f(z)$ if it satisfies $f\left(z_{k}\right)=\zeta$, for each $k$. We say that a complex number $\zeta$ satisfies the Blaschke condition if the $\zeta$-points of $f(z)$ satisfy

$$
\sum_{k=1}^{\infty}\left(1-\left|z_{k}\right|\right)<\infty
$$

Notice that the above condition (1) allows us to define the following Blaschke product (see E. F. Collingwood and A. J. Lohwater [2, p. 28])

$$
B\left(z,\left\{z_{k}\right\}\right)=z^{n} \prod_{k=1}^{\infty} \frac{\left|z_{k}\right|}{z_{k}} \frac{z_{k}-z}{1-\bar{z}_{k} z} \quad\left(z_{k} \neq 0\right) .
$$

In [5, Theorem 1] Lohwater proved that if $f(z)$ is analytic in $D$ and of class (LP), then every complex number $\zeta$ satisfying (1) is an asymptotic value of $f(z)$, i.e. $f(z)$ tends to $\zeta$ along a path terminating at a point on $C$. Naturally, we may ask if $f(z)$ is of class (LP) how many complex numbers can satisfy (1). The answer turns out to be at most one such number and such one number phenomenon can actually occur if the number is 0 as will be seen from the following two results.

Received by the editors October 28, 1980 and, in revised form, February 24, 1981.

1980 Mathematics Subject Classification. Primary 30D40; Secondary 30D50, 30D60.

Key words and phrases. Analytic function, radial limit, Blaschke condition, Lusin-Privaloff's class, boundary behaviour.

'I am indebted to the referee for his many valuable comments in this research. 
THEOREM 1. If $f(z)$ is analytic in D and of class ( $L P)$, then there is at most one number which can satisfy (1).

THEOREM 2. There is a function $f(z)$ analytic in $D$ and of class $(L P)$ such that $f(z)$ has infinitely many zeros which satisfy (1).

2. Normal functions. To prove Theorem 1, we shall need a uniqueness theorem of normal functions. Following O. Lehto and K. I. Virtanen [4, p. 53], we say that a meromorphic function $f(z)$ is normal in $D$ if the family $\left\{f\left(S_{n}(z)\right)\right\}$ is normal in the Montel's sense, where $S_{n}(z)$ denotes an arbitrary one-one mapping from $D$ onto itself. In particular, if $f(z)$ omits three values in $D$, then the family $\left\{f\left(S_{n}(z)\right)\right\}$ is normal due to a well-known theorem of Montel and therefore the function $f(z)$ is normal (see [4, p. 53]). One of the most important boundary properties of normal functions describes that if $f(z)$ is normal in $D$ and has an asymptotic value $v$ at a point $P$ on $C$, then $f(z)$ possesses the angular limit $v$ at $P$ (see [4, Theorem 2]). Based on this property and a theorem of Privaloff (see [2, Theorem 8.1]), we obtain the following uniqueness theorem of normal functions.

LEMMA 1. If $f(z)$ is normal in $D$ and if $E$ is a set of positive linear measure on $C$ such that, for each point $P \in E, f(z)$ has the asymptotic value 0 at $P$, then $f(z)$ is identically zero in $D$.

3. Jordan domains. A domain $G$ is a Jordan domain if its boundary $\partial G$ is a Jordan curve. We say that $G$ is a rectifiable Jordan domain if its boundary $\partial G$ is rectifiable. The key point to prove in Theorem 1 depends on the following basic property between condition (1) and a rectifiable Jordan domain.

Lemma 2. Let $\left\{z_{k}\right\}$ be a sequence of points in D satisfying (1); then for any $\varepsilon$ with $0<\varepsilon<2 \pi$, there is a rectifiable Jordan domain $G \subset D$ such that $G$ contains no points of $z_{k}$ and the linear measure $|\partial G \cap C|>2 \pi-\varepsilon>0$.

Proof. Let $z_{k}=r_{k} e^{i \theta_{k}}$ and $R_{k}=\left\{r e^{i \theta_{k}}: r_{k} \leqslant r \leqslant 1\right\}$; by (1) we can see that the sum of the lengths $\left|R_{k}\right|$ is finite, i.e.

$$
\sum_{k=1}^{\infty}\left|R_{k}\right|=\sum_{k=1}^{\infty}\left(1-\left|z_{k}\right|\right)<\infty .
$$

This gives the idea behind the construction of the desired Jordan domain. Let $G_{k}\left(\varepsilon_{k}\right)$, where $\varepsilon_{k}>0$ will be determined later, be the circular rectangle bounded by two circular arcs $A_{k}, B_{k}$ and two radial segments $S_{k}, T_{k}$ defined by $A_{k}=\left\{r_{k} e^{i \theta}\right.$ : $\left.\theta_{k}-\varepsilon_{k} \leqslant \theta \leqslant \theta_{k}+\varepsilon_{k}\right\}, \quad B_{k}=\left\{e^{i \theta}: \theta_{k}-\varepsilon_{k} \leqslant \theta \leqslant \theta_{k}+\varepsilon_{k}\right\}, \quad S_{k}=\left\{r e^{i\left(\theta_{k}-\varepsilon_{k}\right)}\right.$ : $\left.r_{k} \leqslant r \leqslant 1\right\}$, and $T_{k}=\left\{r e^{i\left(\theta_{k}+\varepsilon_{k}\right)}: r_{k} \leqslant r \leqslant 1\right\}$. Clearly, the closure $\overline{G_{k}\left(\varepsilon_{k}\right)}$ contains $z_{k}$ and $R_{k}$ and is symmetric with respect to $R_{k}$.

We begin with $k=1$ and choose $2 \varepsilon_{1}<\varepsilon / 2$. If the closure $\overline{G_{1}\left(\varepsilon_{1}\right)}$ contains all points of $z_{k}$, then the complement $H_{1}=D-\overline{G_{1}\left(\varepsilon_{1}\right)}$ is a Jordan domain whose boundary $\partial H_{1}$ has length $\left|\partial H_{1}\right|<2 \pi+2\left(1-\left|z_{1}\right|\right)$ and the linear measure of the intersection $\left|\partial H_{1} \cap C\right|=2 \pi-2 \varepsilon_{1}>2 \pi-\varepsilon / 2$. The domain $H_{1}$ is the desired one because it contains no points of $z_{k}$. On the other hand, we may, without loss of 
generality, assume that $z_{2} \in H_{1}$. Choose $2 \varepsilon_{2}<\varepsilon / 4$ so that the closures $\overline{G_{1}\left(\varepsilon_{1}\right)}$ and $\overline{G_{2}\left(\varepsilon_{2}\right)}$ are disjoint. Then the complement $H_{2}=D-G_{1}\left(\varepsilon_{1}\right) \cup G_{2}\left(\varepsilon_{2}\right)$ is again a Jordan domain whose boundary $\partial H_{2}$ satisfies $\left|\partial H_{2}\right|<2 \pi+2\left(1-\left|z_{1}\right|\right)+$ $2\left(1-\left|z_{2}\right|\right)$ and

$$
\left|\partial H_{2} \cap C\right|=2 \pi-2\left(\varepsilon_{1}+\varepsilon_{2}\right)>2 \pi-(\varepsilon / 2+\varepsilon / 4) .
$$

If $H_{2}$ contains no points of $z_{k}$, we are done; otherwise, by continuing this process, we finally obtain a sequence of circular rectangles $\left\{G_{k}\left(\varepsilon_{k}\right)\right\}$, where $2 \varepsilon_{k}<\varepsilon / 2^{k}$, such that the closures $\overline{G_{k}\left(\varepsilon_{k}\right)}$ are all disjoint. Let $G=D-\cup_{k=1}^{\infty} G_{k}\left(\varepsilon_{k}\right)$; then $G$ is a Jordan domain for which

$$
|\partial G|<2 \pi+2 \sum_{k=1}^{\infty}\left(1-\left|z_{k}\right|\right)<\infty
$$

and

$$
|\partial G \cap C|=2 \pi-2 \sum_{k=1}^{\infty} \varepsilon_{k}>2 \pi-\varepsilon>0 .
$$

Since $G$ contains no points of $z_{k}$, we thus conclude the assertion.

4. Proof of Theorem 1. With the help of the above two lemmas, we are now able to prove Theorem 1. Let $f(z)$ be analytic in $D$ and of class (LP). To prove the assertion, we suppose on the contrary that there are two numbers $\zeta_{1}$ and $\zeta_{2}$ that satisfy (1). Denote by $\left\{z_{i n}\right\}$ the $\zeta_{i}$-points for $i=1,2$ respectively. Then the union $\left\{z_{1 n}\right\} \cup\left\{z_{2 n}\right\}$ again satisfies (1). It follows from Lemma 2 that there is a Jordan domain $G \subset D$ such that $G$ contains no points of $\left\{z_{1 n}\right\} \cup\left\{z_{2 n}\right\}$ and whose boundary $\partial G$ is rectifiable and satisfies $|\partial G \cap C|>0$. Let $z=z(w)$ map $D_{w}:|w|<1$ conformally onto $G$. Since $\partial G$ is rectifiable, a theorem of Carathéodory [2, Theorem 3.2] implies that $z(w)$ may be extended to the closure $\overline{D_{w}}$ by defining $z\left(e^{i \theta}\right)$ to be the degenerate cluster set at $e^{i \theta}$, so as to give a mapping of $\overline{D_{w}}$ onto $\bar{G}$, which is a homeomorphism. Let $e^{i \phi} \in \partial G \cap C$ and let $r_{\phi}$ be a radial segment contained in $G$ and ending at the point $e^{i \phi}$. Denote by $w(z)$ the inverse mapping of $z(w)$. Then the image $w\left(r_{\phi}\right)$ is an arc ending at a point $e^{i \theta}$ on $|w|=1$.

We now consider the function $g(w)=f(z(w))$ which is analytic in $D_{w}$. Since the domain $G$ contains no points of $\left\{z_{1 n}\right\} \cup\left\{z_{2 n}\right\}$, it follows that the function $g(w)$ omits the three values $\zeta_{1}, \zeta_{2}$, and $\infty$, so that $g(w)$ is normal in $D_{w}$. In view of the class (LP), we can see that for almost every point $e^{i \phi} \in \partial G \cap C$, the radial limit $f\left(e^{i \phi}\right)=0$. It follows that the function $g(w)$ has the asymptotic value 0 along the arc $w\left(r_{\phi}\right)$ ending at a point $e^{i \theta}$ on $|w|=1$. Since $\partial G$ is rectifiable and $|\partial G \cap C|>0$, by applying a theorem of Riesz (see [2, Theorem 3.3]) we conclude that there is a set $E$ of positive linear measure on $|w|=1$ such that the function $g(w)$ has the asymptotic value 0 at every point of $E$. According to Lemma 1, the function $g(w)$ as well as $f(z)$ must be identically zero, a contradiction. This completes the proof.

5. Proof of the Theorem 2. To prove this theorem, we shall first construct a function $f(z)$ of class (LP) which omits the value 0 . To do this, we let $E$ be a set of first category (i.e. countable union of nowhere dense sets) and of measure $2 \pi$ on 
$|z|=1$. For each $e^{i \theta} \in E$, we let $r_{\theta}$ be the radius ending at $e^{i \theta}$. Denote by $\phi(z)$ a real valued continuous function in $D$ such that the radial $\operatorname{limit}_{\lim _{r \rightarrow 1}} \phi\left(r e^{i \theta}\right)=\infty$ for each $e^{i \theta} \in E$. Then by a theorem of F. Bagemihl and W. Seidel (see [2, Theorem 8.11]), there exists a function $g(z)$ analytic in $D$ such that

$$
\lim _{r \rightarrow 1}\left(g\left(r e^{i \theta}\right)-\phi\left(r e^{i \theta}\right)\right)=0 \quad \text { for each } e^{i \theta} \in E .
$$

This yields for each $e^{i \theta} \in E, \lim _{r \rightarrow 1} \operatorname{Re} g\left(r e^{i \theta}\right)=\infty$ and $\lim _{r \rightarrow 1} \operatorname{Im} g\left(r e^{i \theta}\right)=0$. Let $h(z)=e^{-g(z)}$; then $h(z)$ is analytic in $D$ and has the radial limit 0 at every point $e^{i \theta} \in E$. Since the measure $|E|=2 \pi$, the function $h(z)$ is of class (LP) and omits the value 0 in $D$.

Finally, we let $\left\{z_{k}\right\}$ be a sequence of points in $D$ satisfying (1) and let $B(z)=B\left(z,\left\{z_{k}\right\}\right)$ be the associated Blaschke product defined by (2). Then clearly the function $f(z)=B(z) h(z)$ is of class (LP) and the number 0 satisfies (1). This proves the assertion.

6. Boundary behaviour of class (LP). In [5, Theorem 1], Lohwater has shown that if $f(z)$ is analytic in $D$ and of class (LP), then $f(z)$ admits $\infty$ as an asymptotic value. Let $E$ be the set of all points $e^{i \theta}$ for which $f(z)$ has the asymptotic value $\infty$ at $e^{i \theta}$. Then $E$ is not empty and of linear measure zero. Naturally, we may ask the set theoretical properties of $E$, for instance, whether $E$ is dense, countable, first category, or not. As a consequence of Lohwater's theorem [5, Theorem 2], we obtain the following result which will be needed in the sequel.

THEOREM 3. If $f(z)$ is analytic in D and of class $(L P)$ and if $E$ is the set of all points $e^{i \theta}$ for which $f(z)$ has the asymptotic value $\infty$ at $e^{i \theta}$, then $E$ is dense on $C$.

As usual (see [2, p. 147]), we shall call a point $e^{i \theta}$ a Plessner point of a function $f(z)$ if for every small angle $\Delta$ between pairs of chords through $e^{i \theta}$ the angular cluster set of $f(z)$ over $\Delta$ is the extended plane. Furthermore, we shall call a point $e^{i \theta}$ a Fatou point of a function $f(z)$ if $f(z)$ has an angular limit at $e^{i \theta}$.

THEOREM 4. If $f(z)$ is analytic in $D$ and of class $(L P)$, then almost every point on $C$ is a Plessner point of $f(z)$.

Proof. According to a theorem of Plessner [2, Theorem 8.2], we know that almost every point on $C$ will be either a Fatou point or a Plessner point of $f(z)$. It is sufficient to prove that the set $F(f)$ of all Fatou points of $f(z)$ is of linear measure zero on $C$. Suppose on the contrary that the measure $|F(f)|>0$. Let $E$ be the set of all points $e^{i \theta}$ such that the function $f(z)$ has radial limit 0 at $e^{i \theta}$. Then we have the measure $|E|=2 \pi$ and therefore the measure $|E \cap F(f)|>0$. Since for each point $e^{i \theta} \in E \cap F(f)$ the function $f(z)$ has the angular limit 0 at $e^{i \theta}$, it follows from the aforementioned theorem of Privaloff that $f(z)$ is identically zero, a contradiction. This proves the result.

As usual (see [2, p. 43]), we call a point $e^{i \theta}$ on $C$ a Picard point of an analytic function $f(z)$ defined in $D$ if $f(z)$ assumes infinitely often in any neighborhood of $e^{i \theta}$ all finite complex values with at most one exception. Notice that there are some functions which have no Picard points but which have Plessner points almost 
everywhere on $C$. For instance, the elliptic modular functions, which omit two finite values, do have this property, due to $\mathrm{F}$. Bagemihl [1, Theorem 1].

Also note that if $f(z)$ is meromorphic in $D$, then we call a point $e^{i \theta}$ on $C$ a Picard point of $f(z)$ if $f(z)$ assumes infinitely often in any neighborhood of $e^{i \theta}$ all complex values with at most two exceptions.

THEOREM 5. If $f(z)$ is analytic in $D$ and of class $(L P)$, then every point on $C$ is a Picard point of $f(z)$.

Proof. Suppose on the contrary that there is a point $e^{i \theta}$ on $C$ which is not a Picard point of $f(z)$. Then there is a neighborhood $V$ of $e^{i \theta}$ bounded by an $\operatorname{arc} A$ on $C$ which contains the point $e^{i \theta}$, such that the function $f(z)$ omits two finite values in $V$. Therefore $f(z)$ is normal in $V$, so that $f(z)$ is identically zero, a contradiction.

7. Generalizations of class (LP). Instead of the class (LP) on the whole circle $C$, Lohwater [5, p. 79] has considered a more general case, i.e. an analytic function $f(z)$ is of class (LP) on an arc $A$ of $C$ if $f(z)$ has the radial limit 0 for almost all points on $A$. In [5, Theorem 2], Lohwater has extended his result to the class (LP) on an arc of $C$. In this connection, it is easily seen that the above five theorems can be extended to this more general class and, in fact, such an extension will be an easy consequence of the following theorems.

We now consider a different kind of extension. Instead of analytic functions, we shall consider meromorphic functions. We say that a meromorphic function $f(z)$ is of class (LP, $a$ ), $a \neq \infty$, on an $\operatorname{arc} A$ of $C$ if $f(z)$ has the radial limit $a$ for almost all points on $A$. Furthermore, instead of the whole arc $A$ on $C$, we shall regard only a subset $M$ of $A$ which is metrical dense on $A$, i.e. the measure $|M \cap S|>0$ for any subarc $S$ of $A$. For convenience, we denote by $M(\mathrm{LP}, a, A)$ the class of all nonconstant meromorphic functions $f(z)$ which have the radial limit $a$ at every point on a metrical dense subset of the $\operatorname{arc} A$ on $C$. With this notion, we shall first extend Theorem 1 as follows.

THEOREM 6. If $f(z)$ is meromophic in $D$ and of class $M(L P, a, A)$, then there are at most two numbers which can satisfy (1).

Proof. The argument here is the same as in Theorem 1 and we sketch it. Suppose there are three numbers $\zeta_{i}, i=1,2,3$, satisfying (1); then there is a rectifiable Jordan domain $G \subset D$ for which the function $f(z)$ omits these three values $\zeta_{i}$, $i=1,2,3$ in $G$, so that $f(z)$ is normal in $G$. Since metrical dense sets have the property of positive linear measure on $A$ and such property is conformally invariant with respect to a rectifiable Jordan domain it follows that $f(z)=a$ identically, a contradiction.

Turning to Theorem 2, we have the following extension.

THEOREM 7. If $a$ and $b$ are two arbitrary numbers, then there is a function $f(z)$ in the class $M(L P, a, A)$ which omits $a$ and $b$. 
Proof. Let $h(z)$ be the function defined in Theorem 2 which is of class (LP) and omits both 0 and $\infty$. Then the function

$$
f(z)=(b h(z)-a) /(h(z)-1)
$$

belongs to the class (LP, $a$ ) and omits $a$ and $b$. Clearly, this class (LP, $a$ ) is a subclass of $M(\mathrm{LP}, a, A)$, so that the function $f(z)$ is the desired one.

Unfortunately, we have not been able to extend Theorem 3 in the same way as above. We can prove only the following slight extension.

THEOREM 8. If $f(z)$ is of class $(L P, a)$ on an arc $A$ of $C$ and omits two numbers $a$ and $b$, and if $E$ is the set of all points $e^{i \theta}$ on $A$ for which $f(z)$ has the asymptotic value $b$ at $e^{i \theta}$, then $E$ is dense on $A$.

Proof. The proof is easy. We need only consider the function

$$
g(z)=(f(z)-a) /(f(z)-b)
$$

then the assertion follows from Theorem 3.

As for Theorem 4, it can be easily extended to functions of the class (LP, $a$ ) on an arc of $C$, but we have not been able to extend to the class $M(\mathrm{LP}, a, A)$. On the other hand, it is easy to see that Theorem 5 does hold for this class and we state it without proof as follows.

THEOREM 9. If $f(z)$ is meromorphic in $D$ and of class $M(L P, a, A)$, then every point on $A$ is a Picard point of $f(z)$.

Finally, we shall extend Theorem 5 in a different way. To do this, we shall need the notion of radial Julia points. Following E. F. Collingwood and G. Piranian [3, p. 246], we call a point $e^{i \theta}$ a Julia point of a function $f(z)$ if in every small angle $\Delta$ between pairs of chords through $e^{i \theta}$ the function $f(z)$ assumes all values on the Riemann sphere except possibly two. In particular, we call a point $e^{i \theta}$ a radial Julia point of $f(z)$ if the above property holds for every angle $\Delta$ which meets the radius ending at $e^{i \theta}$. Clearly, each Julia point is a radial Julia point. In [3, Theorem 1], the authors have constructed a function for which each point on $C$ is a Julia point. By the same argument as [3], there can be constructed a function which has radial Julia points, but no Julia points. We omit such a construction and devote ourselves to the study of radial Julia points for functions in the class (LP, $a$ ).

THeORem 10. If $f(z)$ is meromorphic in $D$ and of class $(L P, a)$ on an arc $A$ of $C$, then almost every point on $A$ is a radial Julia point of $f(z)$.

Proof. Let $J(f)$ be the set of all radial Julia points on $A$ and let $K(a)$ be the set of all points $e^{i \theta} \in A$ such that $f(z)$ has the radial limit $a$ at $e^{i \theta}$; then the measure $|K(a)|=|A|$. To prove the assertion $|J(f)|=|A|$, suppose on the contrary that $|J(f)|<|A|$. It follows that the set $E=(A-J(f)) \cap K(a)$ has positive measure 
and at every point $e^{i \theta} \in E$, there is an angle $\Delta_{\theta}(\phi)$ of opening $\phi$ viewing from the point $e^{i \theta}$ such that $\Delta_{\theta}(\phi)$ meets the radius $R_{\theta}$ ending at $e^{i \theta}$ and $f(z)$ omits three values in $\Delta_{\theta}(\phi)$. Since $e^{i \theta} \in K(a)$ and $f(z)$ is normal in $\Delta_{\theta}(\phi)$, the function $f(z)$ tends to $a$ uniformly in a subangle $\Delta$ of $\Delta_{\theta}(\phi)$, so that the function $f(z)-a$ must be bounded in $\Delta$. By choosing a subangle, we may assume that $\Delta_{\theta}(\phi)$ is symmetric with respect to $R_{\theta}$ and the function $f(z)$ is bounded by $2|a|$ in $\Delta_{\theta}(\phi)$.

We now let $E_{n}$ be the subset of $E$ such that for each point $e^{i \theta} \in E_{n}$ the associated angle $\Delta_{\theta}(\phi)$ has opening $\phi \geqslant 1 / n$ and $f(z)$ is bounded in $\Delta_{\theta}(\phi)$. It follows that $E=\cup_{n=1}^{\infty} E_{n}$, so that there is a subset $E_{n}$ of positive measure. Let $G=\cup_{\theta \in E_{n}} \Delta_{\theta}(\phi)$; then we have $\partial G \cap C=E_{n}$. Since $\left|E_{n}\right|>0$, we may choose a component $G_{m}$ of $G$ for which $\left|\partial G_{m} \cap C\right|>0$. Moreover, from the fact that each opening $\phi \geqslant 1 / n$, it is easy to see that the boundary $\partial G_{m}$ is a rectifiable Jordan curve. Since the function $f(z)$ is uniformly bounded by $2|a|$ in $G_{m}$ and has the radial limit $a$ at every point on $\partial G_{m} \cap C$, it follows that $f(z)=a$ identically, a contradiction. This completes the proof.

8. Remarks and problems. To end up this note, let us pose the following five conjectures.

In view of Theorem 1 , we can prove only at most one number $\zeta$ whose $\zeta$-points satisfy (1). However, we believe that the following result should be true.

Conjecture 1. If $f(z)$ is analytic in $D$ and of class (LP), then there should be no numbers $\zeta$ whose $\zeta$-points satisfy (1) except for $\zeta=0$.

It is known [4, p. 54] that if $f(z)$ assumes three values only a finite number of times in $D$ then $f(z)$ is normal in $D$. This result might be extended to the following criterion of normal functions which would yield Theorem 1 immediately.

Conjecture 2. If $f(z)$ is meromorphic in $D$ and if there are three values satisfying (1), then $f(z)$ is normal in $D$.

Let $f(z)$ be a function in the class (LP) and let $F$ be the set of all points $e^{i \theta}$ for which $f(z)$ has the radial limit 0 at $e^{i \theta}$; then the set $F$ must be of first category due to [6]. Denote by $E$ the set of all points $e^{i \theta}$ for which $f(z)$ has the asymptotic value $\infty$ at $e^{i \theta}$. The function $f(z)$ has the same asymptotic property on the sets $E$ and $F$, except for the measures $|E|=0$ and $|F|=2 \pi$. Since the set $F$ is of first category, we should have the following

Conjecture 3. If $f(z)$ is of class $M(\mathrm{LP}, a, A)$, then the set $E$ is dense, uncountable, and of first category on $C$.

In Theorem 4 we have only proved that almost every point on $C$ is a Plessner point of functions in the class (LP). This result would be an immediate consequence of the following

Conjecture 4. If $f(z)$ is of class (LP), then $f(z)$ has no Fatou points.

Finally, the last Theorem 10 should be extended to the following

Conjecture 5. If $f(z)$ is of class (LP), then almost every point on $C$ is a Julia point.

\section{REFERENCES}

1. F. Bagemihl, Some approximation theorems for normal functions, Ann. Acad. Sci. Fenn. Ser. A I Math. 335 (1963), 1-5.

2. E. F. Collingwood and A. J. Lohwater, The theory of cluster sets, Cambridge Univ. Press, New York, 1966 
3. E. F. Collingwood and G. Piranian, Tsuji functions with segments of Julia, Math. Z. 84 (1964), $246-253$.

4. O. Lehto and K. I. Virtanen, Boundary behaviour and normal meromorphic functions, Acta Math. 97 (1957), 47-65.

5. A. J. Lohwater, On the radial limits of analytic functions, Proc. Amer. Math. Soc. 6 (1955), 79-83.

6. N. Lusin and J. Privaloff, Sur l'unicité et la multiplicité des fonctions analytiques, Ann. École Norm. 42 (1925), 143-191.

Institute of Mathematics, Academia Sinica, Taipei, Taiwan 